\title{
Groundwater quality and hydrogeochemical properties of Torbalı Region, Izmir, Turkey
}

\author{
Gokmen Tayfur • Tugba Kirer • Alper Baba
}

Received: 27 July 2007 / Accepted: 6 November 2007 / Published online: 17 January 2008

(C) Springer Science + Business Media B.V. 2007

\begin{abstract}
The large demand for drinking, irrigation and industrial water in the region of Torbalı (Izmir, Turkey) is supplied from groundwater sources. Almost every factory and farm has private wells that are drilled without permission. These cause the depletion of groundwater and limiting the usage of groundwater. This study investigates spatial and temporal change in groundwater quality, relationships between quality parameters, and sources of contamination in Torbalı region. For this purpose, samples were collected from 10 different sampling points chosen according to their geological and hydrogeological properties and location relative to factories, between
\end{abstract}

G. Tayfur

Department of Civil Engineering,

Izmir Institute of Technology,

Gulbahce Campus, Urla,

Izmir 35340, Turkey

e-mail: gokmentayfur@iyte.edu.tr

T. Kirer

Department of Environmental Engineering,

Izmir Institute of Technology,

Gulbahce Campus, Urla,

Izmir 35340, Turkey

\author{
A. Baba $(\bowtie)$ \\ Department of Geological Engineering, \\ Canakkale Onsekiz Mart University, \\ Terzioglu Campus, \\ Canakkale 17020, Turkey \\ e-mail: alperbaba@comu.edu.tr
}

October 2001 and July 2002. Various physical (pH, temperature, EC), chemical (calcium, magnesium, potassium, sodium, chloride, alkalinity, copper, chromium, cadmium, lead, zinc) and organic (nitrate, nitrite, ammonia, COD and cyanide) parameters were monitored. It was observed that the groundwater has bicarbonate alkalinity. Agricultural contamination was determined in the region, especially during the summer. Nitrite and ammonia concentrations were found to be above drinking water standard. Organic matter contamination was also investigated in the study area. COD concentrations were higher than the permissible limits during the summer months of the monitoring period.

Keywords Groundwater quality · Hydrogeochemical . Hydrogeology · Torbal1 · Izmir

\section{Introduction}

Due to water importance, there have been many studies on groundwater quality in various parts of the world (Reijnders et al. 1998; Soltan 1998; Belgiorno and Napoli 2000; Umar and Ahmad 2000; Aslan et al. 2001; Kampbell et al. 2003; Tariq et al. 2004; Wakida and Lerner 2005; $\mathrm{Hu}$ et al. 2005; Leung and Jiao 2006). Reijnders et al. (1998) studied groundwater quality in the Netherlands collecting samples from 600 locations. They found that $\mathrm{NO}_{3}-\mathrm{N}$ and aluminum concentrations were higher than the supposed standard 
in sandy areas. Belgiorno and Napoli (2000) investigated groundwater quality in a rural area of Italy over a 30 month period at 20 sampling points, also studying the impact of atmospheric pollution on water quality. Umar and Ahmad (2000) investigated groundwater quality in parts of Central Ganga Basin, India. They collected water samples from shallow and deep aquifers and analyzed them for major ions and trace elements. Trace element analysis of water from shallow aquifers showed that the concentration of toxic metals was above permissible limits, thus posing a health hazard, while the water from deep aquifers was comparatively free of contamination (Umar and Ahmad 2000).

Turkey is one of the fastest growing countries in the world. Due to its rapid industrialization and urbanization, domestic, hospital and industrial wastes have become a threat to water sources in the country (Afsin 1997; Ekmekçi and Günay 1997; Kaçaroglu and Günay 1997; Karagüzel et al. 1999; Kaçaroglu 1999; Karlık and Kaya 2001; Kumbur et al. 2001; Eryurt and Sekin 2001; Polat and Y1lmaz 2001; Özçelik and Sariiz 2001; Turan et al. 2001; Baba and Ayyıldız 2006). There have also been groundwater quality studies in parts of Izmir region, Turkey (Asaroğlu et al. 1999; Baba and Sözbilir 2001; Baba et al. 2001; Aslan et al. 2001). Aslan et al. (2001) studied groundwater pollution in the Urla and Menemen areas of Izmir. Pesticides and nitrate contamination were investigated in groundwater samples, whereby it was found that nitrate concentration in some of the samples exceeded WHO (World Health Organization) limits. Asaroğlu et al. (1999) assessed groundwater quality in Buca, Konak and Narlidere in Izmir. Groundwater samples from 15 sampling points were examined monthly for 1 year. Their study showed the existence of organic matter, nitrite, and ammonia pollution. Baba et al. (2001) studied groundwater quality in the city of Izmir where collecting samples from 34 locations. They found that lead and cadmium concentrations were higher than U.S. Environmental Protection Agency (US EPA 1993) standards.

The Torbal1 region of Izmir is rich in terms of groundwater resources. It contains agricultural and urban areas and consequently the large demand for drinking and irrigation water is supplied from groundwater. A concentration of industrial complexes also acquires its water demands from groundwater.
Almost every factory and farm in the region has private wells, mostly drilled without permission, causing depletion of the groundwater. These, in turn, cause groundwater quality problems limiting the usage of groundwater.

The objective of this study is to determine temporal and spatial changes in groundwater quality and the relationships between quality parameters and sources of contamination in Torbal1 region. For this purpose, samples were collected from 10 different sampling points chosen according to their geological and hydrogeological properties and proximity to industry. Therefore, the research encompassed a large area of Torbal1. The samples were analyzed for components such as chloride, nitrate, alkalinity, ammonia nitrogen, potassium, sodium, magnesium, calcium, chemical oxygen demand (COD), $\mathrm{pH}$, electrical conductivity (EC), zinc, cadmium, chromium, copper, lead, nitrite and cyanide.

\section{Study area}

Torball, with an area of $600 \mathrm{~km}^{2}$, is one of the most industrialized urban areas in İzmir. The region has rich groundwater resources that are widely used for industrial, agricultural, and drinking purposes. There are many industries, manufacturing motor vehicles, paint, cigarettes, olive oil, textiles, marble, leather, bricks and so on.

As a surface water source, the Fetrek Creek, which is dry in summer, is located in the region. The creek has been used as a wastewater discharge point by industry for many years. In the area, there is no appropriate sewage collection system or solid waste disposal site; therefore, investigation of the water quality was essential.

In the agricultural areas of Torbal1, vegetables (peppers, celery, lettuce, leeks, etc.), grains, and fruits such as peaches and figs are grown. Pesticides and manure are used to protect and fertilize the plants, namely ammonium sulfate, CAN (calcium, ammonium, and nitrate), ammonium nitrate, triple super phosphate, diammonium phosphate, potassium nitrate, and potassium sulfate. Torbalı is also very close to İzmir Harbor and Aydın Highway; therefore, the number of industrial complexes located there is increasing steadily. All these industries need good quality water. 


\section{Geology and hydrogeology of the study area}

Climate is an important factor affecting the potential for contaminant migration from a release source. Mean values for precipitation, evaporation, evapotranspiration, and estimated percolation help to determine the potential for contaminant transport. In the region, average annual rainfall is $756.6 \mathrm{~mm}$, and average annual temperature is $17^{\circ} \mathrm{C}$. The average rainfall in February, March, and April 2002 was measured as $55.2,95.8$, and $58 \mathrm{~mm}$, respectively.

Torbal1 is situated in Western Anatolia (Fig. 1). The Mendres Massif schists and marbles constitute the basement rocks of the study area. The KemalpaşaTorbalı basin is a NE-SW trending depression lying on the tectonic contact between the İzmir-Ankara zone in the west and the Menderes Massif in the east. İzmirAnkara zone cropping out in the west-horst of the basin consists of blocks of limestone and serpentinite mixed in a martix of turbiditic sandstone-shale, while the horst in the east of the basin is made up of schists intercalated with marbles of the Menderes Massif. Two main lithostratigraphic formations, namely Dereköy and Vişneli, are distinguished around the basin. The Dereköy formation is characterized by a succession of fault-controlled alluvial fan showing both fining and coarsening upward sequences. These coarse grained clastic such as sandstone deposits include some lenses of limestone bearing thin coal intercalations. In the lower part of this formation, some limestone olistolites embedded in red clastics are also observed. The Vişneli formation passes gradationary upward into the upper clastic unit. The latter is made up of fine grained lacustrine sediments which contain felsic tuff lenses. The east-bounding fault of the basin has the characteristic of a growth fault which controlled the Miocene sedimentation. The lithologic and structural properties mentioned above are the main criteria for determining the potential and quality of the groundwater in the investigated area (Baba and Sözbilir 2001). The alluvium unit which constitutes Torbalı plain is a kind of unconfined aquifer. The amount of rainfall affects the groundwater level. The regional groundwater flow is in a north to south direction. Torbali
Fig. 1 Geological map of study area and surrounding (modified after Yilmaz et al. 2000)

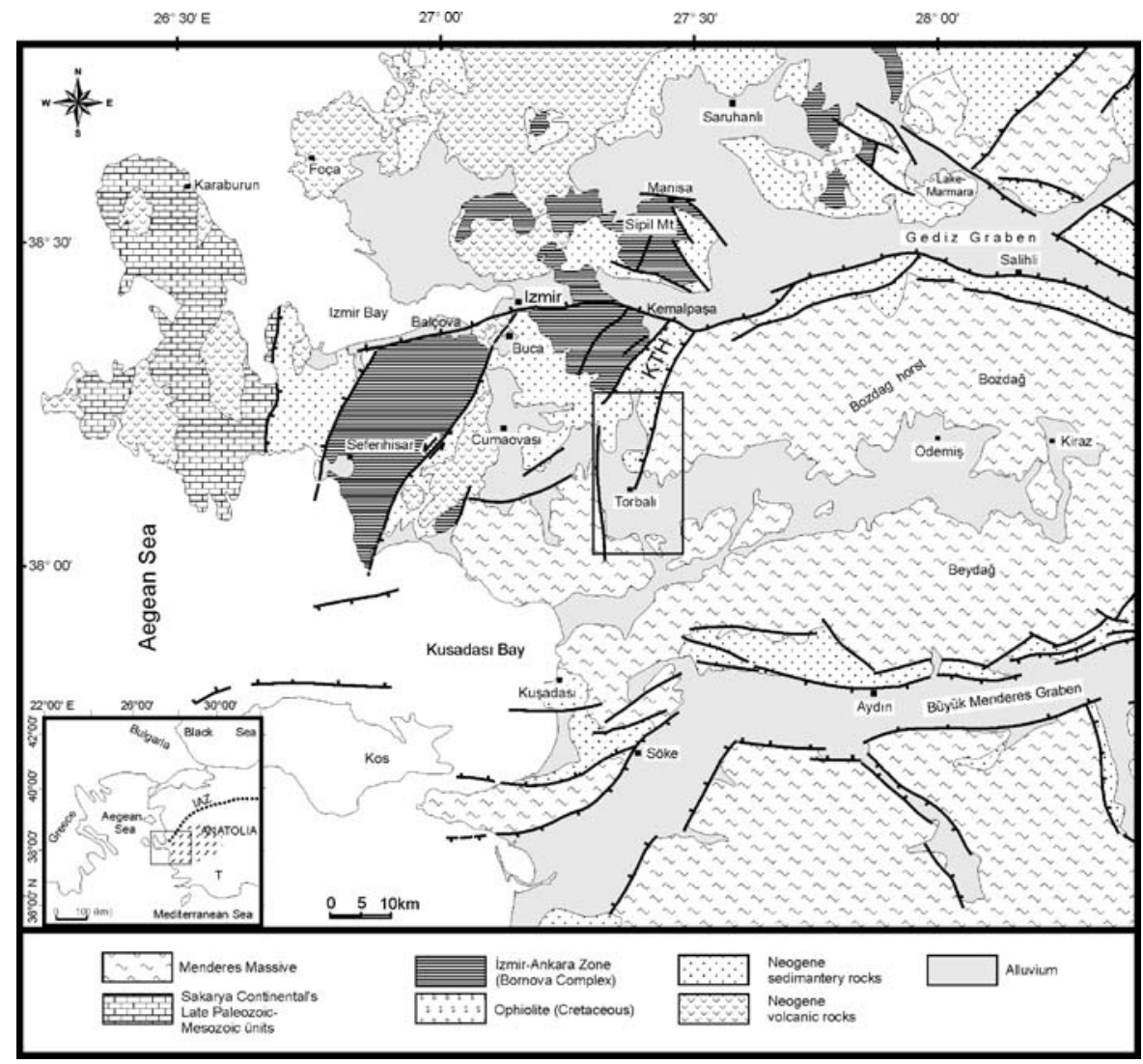


Plain recharges from ground and underground karst sources. The groundwater level in the area varies between 20 and $140 \mathrm{~m}$. Fetrek Creek is a surface water source for the region (Şimşek and Filiz 2001).

\section{Materials and methods}

\section{Sampling}

The procedure for collecting groundwater samples involved the following steps:

- Well evacuation (pumping)

- Sampling

- In-situ (or field analyses); and

- Sample preservation and handling

The groundwater samples were collected using $1 \mathrm{~L}$ polyethylene bottles, labeled to avoid misidentification, and stored at $4^{\circ} \mathrm{C}$ in the refrigerator. Sample preservation was accomplished by adding a nitric acid or base as preservative to adjust $\mathrm{pH}$. Preservatives were added to the container immediately after collecting the samples. Preserving samples in this way retards biodegradation, hydrolysis, precipitation, and sorption reactions.

The sample containers were thoroughly washed with $\mathrm{HNO}_{3}(1+5)$ solution and then rinsed with deionized water before being used for collecting samples for trace elements, nitrate, nitrite, ammonia, chloride, and bicarbonate analysis. Before collecting samples for chemical oxygen demand analysis, the sample containers were washed with $\mathrm{H}_{2} \mathrm{SO}_{4}$ solution and then rinsed with deionized water. Also, each sample container was rinsed with the groundwater sample during the sampling study. Still water in the well prior to sampling may not be representative of in-situ groundwater quality. Therefore, the still water in the well was removed. During sampling, each well was pumped for 5-10 min to prevent contamination through the pipes.

\section{Selection of the observation wells}

The following criteria were considered in the selection of the 10 observation wells to investigate the spatial and temporal change in groundwater quality and hydrogeochemical characteristics of Torbal1 region:

- Distribution of the existing well locations

- Geological and hydrogeological framework of the region; and

- Possible contaminant source areas

Figure 2 presents the location of the selected wells and Fetrek Creek. As seen in Fig. 2, since many industries are located near Fetrek Creek, some observation wells were selected near the creek. The wells denoted $\mathrm{W}-02$ and $\mathrm{W}-05$ are in the industrial
Fig. 2 Location of sampling points in Torbal1, Izmir

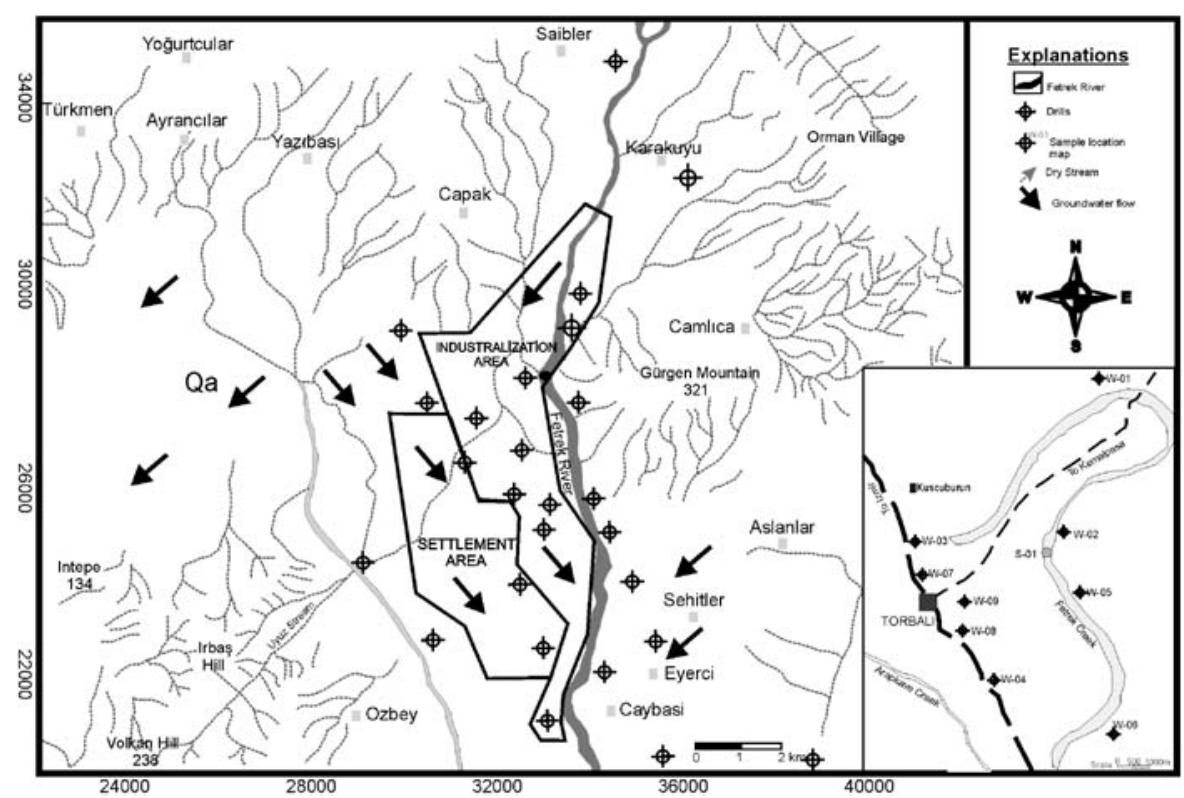


area very close to Fetrek Creek. Other wells denoted W-01 and W-06 are located in the agricultural area which is also near to Fetrek Creek. Wells denoted $\mathrm{W}-03, \mathrm{~W}-04, \mathrm{~W}-07$, and $\mathrm{W}-08$ are located in the center of Torbalı region - some close to gas stations. The well denoted W-09 is used as a municipal water source for Torbal1 and sample S-01 is from Fetrek Creek, a discharge point for industry in the area.

\section{Experimental analysis}

Spectrophotometer reagents were used for the determination of nitrate, ammonia nitrogen, nitrite and cyanide. Determination of alkalinity is based on a titration of the water sample with a strong mineral acid, used as an indicator. The Argentometric method was used for the determination of chloride (AWWA 1995). This method is based on titration of a sample with silver nitrate. Chemical Oxygen Demand (COD) was determined by using colorimetric method (AWWA 1995). Ca, Mg, K, Na, Cd, Cu, Cr, Pb and $\mathrm{Zn}$ were determined by using inductively coupled plasma atomic emission spectrometry (Varian, ICPAES) in Izmir Institute of Technology.

Fecal and total coliforms were analyzed for well $\mathrm{W}-09$, which is one of the municipal water resources in the area. The sample was analyzed within $2 \mathrm{~h}$ right after collection using an appropriate collection technique to analyze microorganisms.

\section{Results and discussion}

Samples were analyzed for the period October 2001 to July 2002. The results are given in Table 1, according to which $\mathrm{pH}$ and $\mathrm{EC}$ values were generally constant during the monitoring period for all the samples. According to TS 266 (Turkish Standards (TS) 2005), pH values must be between 6.5 and 8.5. The $\mathrm{pH}$ values of the samples were within the limits. The wells W-01 and W-06 are used for irrigation, therefore the results of these wells were compared to irrigation water standards. The electrical conductivity (EC) is sensitive to changes in ion concentrations. In the region, EC values were also affected by the changes in ionic species especially by the increasing of bicarbonate and chloride concentration.

At well W-01, the $\mathrm{pH}$ and $\mathrm{EC}$ values were between $7.18-7.58$ and $721-776 \mu \mathrm{S} / \mathrm{cm}$, respectively. Calcium concentrations were about $100 \mathrm{mgL}^{-1}$. Magnesium and sodium concentrations were about 30.0 and $15 \mathrm{mgL}^{-1}$. Potassium concentrations were about $1.5 \mathrm{mgL}^{-1}$. $\mathrm{HCO}_{3}^{-}$and $\mathrm{Cl}^{-}$concentrations were about 250.0 and $35.0 \mathrm{mgL}^{-1}$. $\mathrm{HCO}_{3}^{-}$concentration as $\mathrm{CaCO}_{3}$ was about $210.0 \mathrm{mgL}^{-1}$. Hence, the samples of well W-01 were characterized as hard water. This well is used for irrigation and concentrations of the parameters were within the limits of irrigation water standards.

At well $\mathrm{W}-02, \mathrm{pH}$ values were between the permissible limits and the electrical conductivity values were between 1,401 and $1,765 \mathrm{mS} / \mathrm{cm}$. Calcium concentration was about $155.0 \mathrm{mgL}^{-1}$, which exceeded the TS 266 limit. Magnesium concentrations were about $50.0 \mathrm{mgL}^{-1}$, which is the limit according to TS 266. Sodium concentrations were about $70.0 \mathrm{mgL}^{-1}$, which were within the limits of Turkish Standards. Potassium, $\mathrm{HCO}_{3}^{-}$and $\mathrm{Cl}^{-}$ concentrations were about 2.0, 250 and $330 \mathrm{mgL}^{-1}$ respectively. The $\mathrm{HCO}_{3}^{-}$and $\mathrm{Cl}^{-}$concentrations were observed to change suddenly during the study period. Alkalinity was about $200.0 \mathrm{mgL}^{-1}$, and hence, well W-02 was characterized as hard water. Nitrate concentrations were under the limit of TS 266.

At well W-03, $\mathrm{pH}$ and $\mathrm{EC}$ values were between $7.20-7.69$ and $620-835 \mu \mathrm{S} / \mathrm{cm}$, respectively. Calcium concentration was variable with the lowest value $75.0 \mathrm{mgL}^{-1}$ and the highest value $110.0 \mathrm{mgL}^{-1}$, which exceeded the TS 266 limit. Magnesium concentrations were about $27.0 \mathrm{mgL}^{-1}$ which was also not constant during the monitoring period. Sodium concentrations were about $14.0 \mathrm{mgL}^{-1}$, within the limits of Turkish Standards. Although potassium concentrations were about $1.5 \mathrm{mgL}^{-1}$ in autumn 2001 and winter 2002, it decreased in the summer of 2002. $\mathrm{HCO}_{3}^{-}$concentrations were not constant during the study. $\mathrm{HCO}_{3}^{-}$concentration as $\mathrm{CaCO}_{3}$ showed that the well samples could be characterized as hard water. Although $\mathrm{Cl}^{-}$concentrations were about $40 \mathrm{mgL}^{-1}$, they showed sudden fluctuations. Nitrate concentration exceeded the permissible limit in October 2001.

At well $\mathrm{W}-04, \mathrm{pH}$ and $\mathrm{EC}$ values were between 7.40-7.49 and 684-707 $\mu \mathrm{S} / \mathrm{cm}$, respectively (Table 1). $\mathrm{HCO}_{3}^{-}$concentrations were not constant during the study period. The sample of well W-04 was characterized as hard water. $\mathrm{Cl}^{-}$concentrations were about $40 \mathrm{mgL}^{-1}$, within permissible limits. The concen- 
Table 1 Chemical properties of water in Torbalı Basin

\begin{tabular}{|c|c|c|c|c|c|c|c|c|c|c|c|c|c|c|}
\hline \multirow[t]{2}{*}{ Location } & \multicolumn{10}{|l|}{ W-01 } & \multicolumn{4}{|l|}{ W-02 } \\
\hline & $\begin{array}{l}\text { October } \\
2001\end{array}$ & $\begin{array}{l}\text { November } \\
2001\end{array}$ & $\begin{array}{l}\text { December } \\
2001\end{array}$ & $\begin{array}{l}\text { January } \\
2002\end{array}$ & $\begin{array}{l}\text { February } \\
2002\end{array}$ & $\begin{array}{l}\text { March } \\
2002\end{array}$ & $\begin{array}{l}\text { April } \\
2002\end{array}$ & $\begin{array}{l}\text { May } \\
2002\end{array}$ & $\begin{array}{l}\text { June } \\
2002\end{array}$ & $\begin{array}{l}\text { July } \\
2002\end{array}$ & $\begin{array}{l}\text { October } \\
2001\end{array}$ & $\begin{array}{l}\text { November } \\
2001\end{array}$ & $\begin{array}{l}\text { December } \\
2001\end{array}$ & $\begin{array}{l}\text { January } \\
2002\end{array}$ \\
\hline $\mathrm{t}^{\circ} \mathrm{C}$ & & & & 18 & 17 & 16 & 19 & 20 & 19 & 18 & & & & 18 \\
\hline $\mathrm{pH}$ & & & 7.2 & 7.22 & 7.58 & 7.18 & 7.43 & 7.18 & 7.45 & 7.4 & & & 7.23 & 7.1 \\
\hline $\mathrm{EC}(\mu \mathrm{S} / \mathrm{cm})$ & & & & 766 & 771 & 754 & 726 & 735 & 730 & 721 & & & & 1742 \\
\hline $\mathrm{HCO}_{3}\left(\mathrm{mgL}^{-1}\right)$ & & 268.4 & 311.1 & 214.72 & 217.6 & 274.5 & 251.3 & 252.54 & 292.8 & 292.8 & & 244 & 268.4 & 211.06 \\
\hline $\mathrm{Cl}\left(\mathrm{mgL}^{-1}\right)$ & & & 32.5 & 38 & 41 & 38 & 35 & 37 & 45 & 37 & & & 315.9 & 355 \\
\hline $\mathrm{Ca}\left(\mathrm{mgL}^{-1}\right)$ & & 109 & 103 & 103 & 104 & 103 & 99 & 100 & 101 & 100 & & 164 & 150 & 173 \\
\hline $\operatorname{Mg}\left(\mathrm{mgL}^{-1}\right)$ & & 32 & 30 & 31 & 26 & 30 & 28 & 29 & 28 & 34 & & 54 & 31 & 61 \\
\hline $\mathrm{Na}\left(\mathrm{mgL}^{-1}\right)$ & & 15 & 19 & 16 & 15 & 18 & 15 & 14 & 15 & 14 & & 43 & 39 & 75 \\
\hline $\mathrm{K}\left(\mathrm{mgL}^{-1}\right)$ & & 2 & 2 & 2 & 2 & 2 & 2 & 1.5 & 1 & 1.5 & & 2 & 2.5 & 2.5 \\
\hline $\mathrm{NO}_{3}-\mathrm{N}\left(\mathrm{mgL}^{-1}\right)$ & 11 & 5 & 7 & 9 & 9 & 7 & 4.5 & 2 & 7 & 5 & 6 & 2 & 2 & 2 \\
\hline $\mathrm{NO}_{3}^{-}\left(\mathrm{mgL}^{-1}\right)$ & 48 & 23.5 & 29 & 40 & 40.7 & 32 & 19.8 & 10.75 & 26 & 28.4 & 24.5 & 6.2 & 9.8 & 8.3 \\
\hline $\mathrm{NO}_{2}-\mathrm{N}\left(\mathrm{mgL}^{-1}\right)$ & 2 & 1 & 2 & 1 & 2 & & & & & & 1 & 1 & 2 & 3 \\
\hline $\mathrm{NH}_{3}-\mathrm{N}\left(\mathrm{mgL}^{-1}\right)$ & & 0.05 & 0.02 & & 0 & 0 & 0.02 & 0.01 & 0.03 & 0.03 & & 0.17 & 0.05 & \\
\hline $\operatorname{COD}\left(\mathrm{mgL}^{-1}\right)$ & 17 & 2 & 0 & 4 & 0 & & & & & & 122 & 3 & 0 & 5 \\
\hline Cyanide $\left(\mathrm{mgL}^{-1}\right)$ & & 0.05 & 0.02 & & 0 & 0 & 0.02 & 0.01 & 0.03 & 0.03 & & 0.17 & 0.05 & \\
\hline $\mathrm{CaCO}_{3}\left(\mathrm{mgL}^{-1}\right)$ & & 220 & 255 & 176 & 178 & 225 & 206 & 207 & 240 & 240 & - & 200 & 220 & 173 \\
\hline
\end{tabular}

\begin{tabular}{|c|c|c|c|c|c|c|c|c|c|c|c|c|c|}
\hline \multirow[t]{2}{*}{ Location } & \multicolumn{6}{|l|}{$\mathrm{W}-02$} & \multicolumn{7}{|l|}{$\mathrm{W}-03$} \\
\hline & $\begin{array}{l}\text { February } \\
2002\end{array}$ & $\begin{array}{l}\text { March } \\
2002\end{array}$ & $\begin{array}{l}\text { April } \\
2002\end{array}$ & $\begin{array}{l}\text { May } \\
2002\end{array}$ & $\begin{array}{l}\text { June } \\
2002\end{array}$ & $\begin{array}{l}\text { July } \\
2002\end{array}$ & $\begin{array}{l}\text { October } \\
2001\end{array}$ & $\begin{array}{l}\text { November } \\
2001\end{array}$ & $\begin{array}{l}\text { December } \\
2001\end{array}$ & $\begin{array}{l}\text { January } \\
2002\end{array}$ & $\begin{array}{l}\text { February } \\
2002\end{array}$ & $\begin{array}{l}\text { March } \\
2002\end{array}$ & $\begin{array}{l}\text { April } \\
2002\end{array}$ \\
\hline $\mathrm{t}^{\circ} \mathrm{C}$ & 16 & 13 & 18 & 18 & 20 & 18 & & & & 16 & 16 & 12 & 17 \\
\hline $\mathrm{pH}$ & 7.22 & 7.21 & 7.55 & 7.24 & 7.42 & 7.43 & & & 7.35 & 7.5 & 7.49 & 7.52 & 7.61 \\
\hline $\mathrm{EC}(\mu \mathrm{S} / \mathrm{cm})$ & 1765 & 1725 & 1586 & 1608 & 1531 & 1401 & & & & 680 & 627 & 622 & 624 \\
\hline $\mathrm{HCO}_{3}\left(\mathrm{mgL}^{-1}\right)$ & 219.6 & 274.5 & 253.8 & 248.9 & 292.8 & 280.6 & & 219.60 & 256.20 & 186.66 & 189.10 & 244.00 & 219.6 \\
\hline $\mathrm{Cl}\left(\mathrm{mgL}^{-1}\right)$ & 352.5 & 340 & 307 & 339 & 325 & 270 & & & 51.0 & 41.5 & 32.5 & 40.0 & 30.0 \\
\hline $\mathrm{Ca}\left(\mathrm{mgL}^{-1}\right)$ & 164 & 158 & - & 158 & 160 & 142 & & 104 & 92 & 93 & 102 & 82 & 75 \\
\hline $\mathrm{Mg}\left(\mathrm{mgL}^{-1}\right)$ & 48 & 52 & - & 53 & 54 & 50 & & 32 & 32 & 30 & 19 & 26 & 25 \\
\hline $\mathrm{Na}\left(\mathrm{mgL}^{-1}\right)$ & 75 & 77 & - & 80 & 63 & 61 & & 12 & 22 & 18 & 14 & 13 & 16 \\
\hline $\mathrm{K}\left(\mathrm{mgL}^{-1}\right)$ & 2 & 1.5 & - & 2 & 2 & 2 & & 1.5 & 1.5 & 1.5 & 2.0 & 1.0 & 0.5 \\
\hline $\mathrm{NO}_{3}-\mathrm{N}\left(\mathrm{mgL}^{-1}\right)$ & 2 & 1.5 & 2 & 4 & 5 & 4 & 13 & 7 & 8 & 7 & 6 & 4 & 3 \\
\hline $\mathrm{NO}_{3}^{-}\left(\mathrm{mgL}^{-1}\right)$ & 8.6 & 6 & 8.7 & 17.5 & 18.1 & 17 & 58.0 & 31.30 & 34.5 & 30 & 27.6 & 39100 & 39126 \\
\hline $\mathrm{NO}_{2}-\mathrm{N}\left(\mathrm{mgL}^{-1}\right)$ & 1 & & & & & & 2.0 & 1.0 & 2.0 & 1.0 & 27.6 & & \\
\hline $\mathrm{NH}_{3}-\mathrm{N}\left(\mathrm{mgL}^{-1}\right)$ & 0.04 & 0.03 & 0.02 & 0.02 & 0.01 & 0.01 & & 0.07 & 0.02 & & 0.06 & 0 & 0 \\
\hline $\operatorname{COD}\left(\mathrm{mgL}^{-1}\right)$ & 56 & & & & & & 44 & 15 & 2 & 1 & 0 & & \\
\hline Cyanide $\left(\mathrm{mgL}^{-1}\right)$ & 0.04 & 0.03 & 0.02 & 0.02 & 0.01 & 0.01 & & 0.07 & 0.02 & & 0.06 & 0 & 0 \\
\hline $\mathrm{CaCO}_{3}\left(\mathrm{mgL}^{-1}\right)$ & 180 & 225 & 208 & 204 & 240 & 230 & - & 180 & 210 & 153 & 155 & 200 & 180 \\
\hline
\end{tabular}

\begin{tabular}{|c|c|c|c|c|c|c|c|c|c|c|c|c|c|c|}
\hline \multirow[t]{2}{*}{ Location } & \multicolumn{3}{|l|}{ W-03 } & \multicolumn{4}{|l|}{ W-04 } & \multicolumn{7}{|l|}{ W-05 } \\
\hline & $\begin{array}{l}\text { May } \\
2002\end{array}$ & $\begin{array}{l}\text { June } \\
2002\end{array}$ & $\begin{array}{l}\text { July } \\
2002\end{array}$ & $\begin{array}{l}\text { April } \\
2002\end{array}$ & $\begin{array}{l}\text { May } \\
2002\end{array}$ & $\begin{array}{l}\text { June } \\
2002\end{array}$ & $\begin{array}{l}\text { July } \\
2002\end{array}$ & $\begin{array}{l}\text { October } \\
2001\end{array}$ & $\begin{array}{l}\text { November } \\
2001\end{array}$ & $\begin{array}{l}\text { December } \\
2001\end{array}$ & $\begin{array}{l}\text { January } \\
2002\end{array}$ & $\begin{array}{l}\text { February } \\
2002\end{array}$ & $\begin{array}{l}\text { March } \\
2002\end{array}$ & $\begin{array}{l}\text { April } \\
2002\end{array}$ \\
\hline $\mathrm{t}^{\circ} \mathrm{C}$ & 19 & 20 & 22 & 22 & 21 & 24 & 25 & & & & 14 & 15 & 14 & 15 \\
\hline $\mathrm{pH}$ & 7.57 & 7.69 & 7.2 & 7.47 & 7.45 & 7.49 & 7.4 & & & 7.35 & 7.64 & 7.48 & 7.56 & 7.49 \\
\hline $\mathrm{EC}(\mu \mathrm{S} / \mathrm{cm})$ & 632 & 799 & 835 & 684 & 685 & 707 & 690 & & & & 920 & 1124 & 1013 & 808 \\
\hline $\mathrm{HCO}_{3}\left(\mathrm{mgL}^{-1}\right)$ & 213.0 & 292.8 & 305.0 & 246.4 & 229.4 & 287.9 & 280.6 & & 451.40 & 423.34 & 209.84 & 224.48 & 250.10 & 219.6 \\
\hline $\mathrm{Cl}\left(\mathrm{mgL}^{-1}\right)$ & 45.0 & 65.0 & 53.0 & 32.0 & 47.5 & 45.0 & 32.0 & & & 246.9 & 104.5 & 152.5 & 140.0 & 78.5 \\
\hline $\mathrm{Ca}\left(\mathrm{mgL}^{-1}\right)$ & 87 & 109 & 106 & 90 & 92 & 94 & 92 & & 194 & 49 & 39 & 72 & 68 & 61 \\
\hline $\mathrm{Mg}\left(\mathrm{mgL}^{-1}\right)$ & 25 & 34 & 36 & 28 & 28 & 30 & 30 & & 57 & 12 & 11 & 19 & 20 & 18 \\
\hline $\mathrm{Na}\left(\mathrm{mgL}^{-1}\right)$ & 14 & 13 & 12 & 14 & 16 & 14 & 12 & & 265 & 150 & 104 & 95 & 88 & 68 \\
\hline $\mathrm{K}\left(\mathrm{mgL}^{-1}\right)$ & 0.5 & 1.0 & 0.5 & 1.0 & 1.0 & 0.5 & 1.0 & & 5.0 & 5 & 3.0 & 3.5 & 4.0 & 3.5 \\
\hline $\mathrm{NO}_{3}-\mathrm{N}\left(\mathrm{mgL}^{-1}\right)$ & 3 & 8 & 8 & 6 & 5 & 9 & 6 & 4 & 3 & 3 & 3 & 2.5 & 1 & 1 \\
\hline $\mathrm{NO}_{3}^{-}\left(\mathrm{mgL}^{-1}\right)$ & 15.0 & 32.0 & 34.4 & 24.0 & 24.0 & 34.6 & 30.8 & 18.0 & 12.1 & 12.1 & 14.5 & 11.7 & 2 & 5 \\
\hline $\mathrm{NO}_{2}-\mathrm{N}\left(\mathrm{mgL}^{-1}\right)$ & & & & & & & & 1.0 & 1.0 & 2.0 & 1.0 & - & & \\
\hline $\mathrm{NH}_{3}-\mathrm{N}\left(\mathrm{mgL}^{-1}\right)$ & 0.01 & 0 & 0 & 0.01 & 0 & 0.02 & 0.02 & & 0.11 & 0.06 & & 0.05 & 0.03 & 0.05 \\
\hline $\operatorname{COD}\left(\mathrm{mgL}^{-1}\right)$ & & & & & & & & 124 & 10 & 4 & 4 & 44 & & \\
\hline Cyanide $\left(\mathrm{mgL}^{-1}\right)$ & 0.01 & 0 & 0 & 0.01 & 0 & 0.02 & 0.02 & & 0.11 & 0.06 & & 0.05 & 0.03 & 0.05 \\
\hline $\mathrm{CaCO}_{3}\left(\mathrm{mgL}^{-1}\right)$ & 175 & 240 & 250 & 202 & 188 & 236 & 230 & - & 370 & 347 & 172 & 184 & 205 & 180 \\
\hline
\end{tabular}


Table 1 (Continued)

\begin{tabular}{|c|c|c|c|c|c|c|c|c|c|c|c|c|c|}
\hline \multirow[t]{2}{*}{ Location } & \multicolumn{3}{|l|}{ W-05 } & \multicolumn{7}{|l|}{ W-06 } & \multicolumn{3}{|l|}{ W-07 } \\
\hline & $\begin{array}{l}\text { May } \\
2002\end{array}$ & $\begin{array}{l}\text { June } \\
2002\end{array}$ & $\begin{array}{l}\text { July } \\
2002\end{array}$ & $\begin{array}{l}\text { January } \\
2002\end{array}$ & $\begin{array}{l}\text { February } \\
2002\end{array}$ & $\begin{array}{l}\text { March } \\
2002\end{array}$ & $\begin{array}{l}\text { April } \\
2002\end{array}$ & $\begin{array}{l}\text { May } \\
2002\end{array}$ & $\begin{array}{l}\text { June } \\
2002\end{array}$ & $\begin{array}{l}\text { July } \\
2002\end{array}$ & $\begin{array}{l}\text { October } \\
2001\end{array}$ & $\begin{array}{l}\text { November } \\
2001\end{array}$ & $\begin{array}{l}\text { December } \\
2001\end{array}$ \\
\hline $\mathrm{t}^{\circ} \mathrm{C}$ & 15 & 16 & 18 & - & 19 & 21 & 20 & 20 & 21 & 20 & & & \\
\hline $\mathrm{pH}$ & 7.76 & 7.68 & 7.1 & 7.07 & 7.3 & 7.21 & 7.4 & 7.42 & 7.25 & 7.46 & & & 7.19 \\
\hline $\mathrm{EC}(\mu \mathrm{S} / \mathrm{cm})$ & 879 & 935 & 1070 & - & 840 & 838 & 815 & 1039 & 806 & 856 & & & \\
\hline $\mathrm{HCO}_{3}\left(\mathrm{mgL}^{-1}\right)$ & 202.0 & 256.2 & 268.4 & - & 268.40 & 305.00 & 268.4 & 292.8 & 309.9 & 317.2 & & 292.80 & 357.46 \\
\hline $\mathrm{Cl}\left(\mathrm{mgL}^{-1}\right)$ & 130.0 & 120.0 & 155.0 & - & 65.0 & 77.5 & 105.5 & 135.0 & 80.0 & 90.0 & & & 48.5 \\
\hline $\mathrm{Ca}\left(\mathrm{mgL}^{-1}\right)$ & 76 & 79 & 83 & - & - & 100 & 118 & 127 & 101 & 105 & & 129 & 108 \\
\hline $\operatorname{Mg}\left(\mathrm{mgL}^{-1}\right)$ & 22 & 23 & 25 & - & - & 33 & 38 & 41 & 33 & 38 & & 40 & 21 \\
\hline $\mathrm{Na}\left(\mathrm{mgL}^{-1}\right)$ & 70 & 77 & 84 & - & - & 22 & 25 & 26 & 23 & 25 & & 19 & 11 \\
\hline $\mathrm{K}\left(\mathrm{mgL}^{-1}\right)$ & 5 & 3.0 & 3.0 & - & - & - & 1.0 & 1.0 & 1.0 & 1.0 & & 1.5 & 1.5 \\
\hline $\mathrm{NO}_{3}-\mathrm{N}\left(\mathrm{mgL}^{-1}\right)$ & 1 & 2.5 & 2 & - & 6 & 4.5 & 3 & 2.5 & 3 & 2.5 & 7 & 11 & 12 \\
\hline $\mathrm{NO}_{3}^{-}\left(\mathrm{mgL}^{-1}\right)$ & 3.8 & 7.6 & 8.1 & 24.1 & 29.6 & 19 & 12.4 & 13.8 & 11.3 & 16.1 & 30.0 & 48.5 & 53.5 \\
\hline $\mathrm{NO}_{2}-\mathrm{N}\left(\mathrm{mgL}^{-1}\right)$ & & & & & & & & & & & 1.0 & 1.0 & 1.0 \\
\hline $\mathrm{NH}_{3}-\mathrm{N}\left(\mathrm{mgL}^{-1}\right)$ & 0.01 & 0.01 & 0.01 & & 0.02 & 0.03 & 0 & 0 & 0.06 & 0.05 & & 0.08 & 0.05 \\
\hline $\mathrm{COD}\left(\mathrm{mgL}^{-1}\right)$ & & & & 1 & 85 & & & & & & 2 & 0 & 0 \\
\hline Cyanide $\left(\mathrm{mgL}^{-1}\right)$ & 0.01 & 0.01 & 0.01 & 0.02 & & 0.03 & 0 & 0 & 0.06 & 0.05 & & 0.08 & 0.05 \\
\hline $\mathrm{CaCO}_{3}\left(\mathrm{mgL}^{-1}\right)$ & 166 & 210 & 220 & & 220 & 250 & 220 & 240 & 254 & 260 & - & 240 & 293 \\
\hline
\end{tabular}

\begin{tabular}{|c|c|c|c|c|c|c|c|c|c|c|c|c|c|}
\hline \multirow[t]{3}{*}{ Location } & \multicolumn{7}{|l|}{ W-07 } & \multicolumn{6}{|l|}{ W-08 } \\
\hline & January & February & March & April & May & June & July & February & March & April & May & June & July \\
\hline & 2002 & 2002 & 2002 & 2002 & 2002 & 2002 & 2002 & 2002 & 2002 & 2002 & 2002 & 2002 & 2002 \\
\hline $\mathrm{t}^{\circ} \mathrm{C}$ & 15 & 18 & 17 & 19 & 20 & 21 & 21 & 15 & 16 & 19 & 20 & 24 & 21 \\
\hline $\mathrm{pH}$ & 7.31 & 7.29 & 7.16 & 7.4 & 7.42 & 7.3 & 7.6 & 7.3 & 7.4 & 7.4 & 7.39 & 7.37 & 7.5 \\
\hline $\mathrm{EC}(\mu \mathrm{S} / \mathrm{cm})$ & 895 & 892 & 883 & 859 & 835 & 884 & 871 & 867 & 863 & 857 & 726 & 819 & 783 \\
\hline $\mathrm{HCO}_{3}\left(\mathrm{mgL}^{-1}\right)$ & 256.20 & 292.80 & 323.30 & 292.8 & 335.5 & 336.7 & 341.6 & 256.20 & 329.40 & 292.8 & 341.0 & 317.2 & 285.5 \\
\hline $\mathrm{Cl}\left(\mathrm{mgL}^{-1}\right)$ & 54.0 & 47.5 & 50.0 & 42.0 & 57.5 & 52.5 & 57.0 & 40.0 & 52.5 & 44.5 & 57.5 & 35.0 & 47.0 \\
\hline $\mathrm{Ca}\left(\mathrm{mgL}^{-1}\right)$ & 125 & 114 & 115 & 115 & 111 & 115 & 119 & - & 108 & 114 & 115 & 106 & 106 \\
\hline $\mathrm{Mg}\left(\mathrm{mgL}^{-1}\right)$ & 39 & 30 & 35 & 35 & 34 & 35 & 39 & - & 35 & 36 & 35 & 32 & 34 \\
\hline $\mathrm{Na}\left(\mathrm{mgL}^{-1}\right)$ & 15 & 18 & 19 & 20 & 22 & 18 & 19 & - & 18 & 19 & 23 & 18 & 15 \\
\hline $\mathrm{K}\left(\mathrm{mgL}^{-1}\right)$ & 1.5 & 1.5 & 1.5 & 1.0 & 1.0 & 1.0 & 1.0 & - & 1.5 & 1.5 & 1.0 & 1.0 & 1.0 \\
\hline $\mathrm{NO}_{3}-\mathrm{N}\left(\mathrm{mgL}^{-1}\right)$ & 10 & 15 & 12 & 8.5 & 8 & 9 & 11 & 8 & 11 & 7 & 7.5 & 9 & 7.5 \\
\hline $\mathrm{NO}_{3}^{-}\left(\mathrm{mgL}^{-1}\right)$ & 46.0 & 64.6 & 52.2 & 37.0 & 35.5 & 36.3 & 48.3 & 36.6 & 48.0 & 32.0 & 34.0 & 37.1 & 45.0 \\
\hline $\mathrm{NO}_{2}-\mathrm{N}\left(\mathrm{mgL}^{-1}\right)$ & 1.0 & - & & & & & & 1.0 & & & & & \\
\hline $\mathrm{NH}_{3}-\mathrm{N}\left(\mathrm{mgL}^{-1}\right)$ & 0.09 & & 0.03 & 0.01 & 0.02 & 0.03 & 0.03 & 0.08 & 0.02 & 0 & 0.02 & 0.01 & 0.02 \\
\hline $\mathrm{COD}\left(\mathrm{mgL}^{-1}\right)$ & 4 & 3 & & & & & & 4 & & & & & \\
\hline Cyanide $\left(\mathrm{mgL}^{-1}\right)$ & & 0.09 & 0.03 & 0.01 & 0.02 & 0.03 & 0.03 & 0.08 & 0.02 & 0 & 0.02 & 0.01 & 0.02 \\
\hline $\mathrm{CaCO}_{3}\left(\mathrm{mgL}^{-1}\right)$ & 210 & 240 & 265 & 240 & 275 & 276 & 280 & 175 & 270 & 240 & 280 & 260 & 234 \\
\hline
\end{tabular}

\begin{tabular}{|c|c|c|c|c|c|c|c|c|c|c|c|c|c|}
\hline \multirow[t]{2}{*}{ Location } & \multicolumn{10}{|l|}{ W-09 } & \multicolumn{3}{|l|}{ S-01 } \\
\hline & $\begin{array}{l}\text { October } \\
2001\end{array}$ & $\begin{array}{l}\text { November } \\
2001\end{array}$ & $\begin{array}{l}\text { December } \\
2001\end{array}$ & $\begin{array}{l}\text { January } \\
2002\end{array}$ & $\begin{array}{l}\text { February } \\
2002\end{array}$ & $\begin{array}{l}\text { March } \\
2002\end{array}$ & $\begin{array}{l}\text { April } \\
2002\end{array}$ & $\begin{array}{l}\text { May } \\
2002\end{array}$ & $\begin{array}{l}\text { June } \\
2002\end{array}$ & $\begin{array}{l}\text { July } \\
2002\end{array}$ & $\begin{array}{l}\text { October } \\
2001\end{array}$ & $\begin{array}{l}\text { November } \\
2001\end{array}$ & $\begin{array}{l}\text { December } \\
2001\end{array}$ \\
\hline $\mathrm{t}^{\circ} \mathrm{C}$ & & & & 14 & 17 & 19 & 18 & 18 & 18 & 19 & & & \\
\hline $\mathrm{pH}$ & & & 7.47 & 7.52 & 7.3 & 7.37 & 7.51 & 7.33 & 7.48 & 7.16 & & & 8 \\
\hline $\mathrm{EC}(\mu \mathrm{S} / \mathrm{cm})$ & & & & 725 & 713 & 754 & 702 & 696 & 705 & 682 & & & \\
\hline $\mathrm{HCO}_{3}\left(\mathrm{mgL}^{-1}\right)$ & & 292.80 & 284.26 & 229.36 & 280.60 & 292.80 & 256.2 & 311.0 & 268.4 & 219.6 & & 292.80 & 189.10 \\
\hline $\mathrm{Cl}\left(\mathrm{mgL}^{-1}\right)$ & & & 39.0 & 36.0 & 50.0 & 37.5 & 32.0 & 47.5 & 37.0 & 35.0 & & & 33.5 \\
\hline $\mathrm{Ca}\left(\mathrm{mgL}^{-1}\right)$ & & 101 & 94 & 100 & 93 & 94 & 96 & 97 & 95 & 93 & & 43 & 42 \\
\hline $\mathrm{Mg}\left(\mathrm{mgL}^{-1}\right)$ & & 25 & 15 & 29 & 27 & 27 & 27 & 28 & 28 & 30 & & 12 & 5 \\
\hline $\mathrm{Na}\left(\mathrm{mgL}^{-1}\right)$ & & 14 & 9 & 14 & 16 & 16 & 14 & 17 & 13 & 14 & & 16 & 28 \\
\hline $\mathrm{K}\left(\mathrm{mgL}^{-1}\right)$ & & 1.5 & 1.5 & 1.5 & 1.0 & 1.0 & 2.0 & 1.0 & 0.5 & 0.5 & & 4.5 & 2.0 \\
\hline $\mathrm{NO}_{3}-\mathrm{N}\left(\mathrm{mgL}^{-1}\right)$ & 5 & 6 & 6 & 6.5 & 14 & 7 & 5 & 6 & 8 & & 9 & 3 & 2 \\
\hline $\mathrm{NO}_{3}^{-}\left(\mathrm{mgL}^{-1}\right)$ & 22.5 & 26.0 & 28.1 & 29.7 & 62.0 & 30.3 & 20.7 & 26.0 & 33.0 & 33.1 & 36.5 & 12.0 & 9.3 \\
\hline $\mathrm{NO}_{2}-\mathrm{N}\left(\mathrm{mgL}^{-1}\right)$ & 2.0 & 1.0 & 2.0 & 1.0 & 2.0 & & & & & & 10.0 & 9.0 & 8.0 \\
\hline $\mathrm{NH}_{3}-\mathrm{N}\left(\mathrm{mgL}^{-1}\right)$ & & 0.05 & 0 & & 0.08 & 0.03 & 0.07 & 0.05 & 0.02 & 0.03 & & 0.16 & 0.23 \\
\hline $\operatorname{COD}\left(\mathrm{mgL}^{-1}\right)$ & 4 & 1 & 1 & 8 & 1 & & & & & & 482 & 36 & 16 \\
\hline Cyanide $\left(\mathrm{mgL}^{-1}\right)$ & & 0.05 & 0 & & 0.08 & 0.03 & 0.07 & 0.05 & 0.02 & 0.03 & & 0.16 & 0.23 \\
\hline $\mathrm{CaCO}_{3}\left(\mathrm{mgL}^{-1}\right)$ & - & 240 & 198 & 188 & 210 & 240 & 210 & 255 & 220 & 180 & - & 240 & 155 \\
\hline
\end{tabular}


Table 1 (Continued)

\begin{tabular}{|c|c|c|c|c|c|c|c|}
\hline \multirow[t]{2}{*}{ Location } & \multicolumn{7}{|l|}{ S-01 } \\
\hline & January 2002 & February 2002 & March 2002 & April 2002 & May 2002 & June 2002 & July 2002 \\
\hline $\mathrm{t}^{\circ} \mathrm{C}$ & 12 & 10 & 11 & 22 & 25 & 28 & 27 \\
\hline $\mathrm{pH}$ & 8.3 & 8.33 & 8.3 & 8.46 & 8.5 & 8.46 & 8.45 \\
\hline $\mathrm{EC}(\mu \mathrm{S} / \mathrm{cm})$ & 1100 & 715 & 527 & 758 & 2840 & 5430 & 7220 \\
\hline $\mathrm{HCO}_{3}\left(\mathrm{mgL}^{-1}\right)$ & 219.60 & 213.50 & 228.14 & 170.8 & 262.8 & 341.6 & 341.6 \\
\hline $\mathrm{Cl}\left(\mathrm{mgL}^{-1}\right)$ & 150.0 & 60.0 & 32.5 & 62.0 & 640 & 240 & 450 \\
\hline $\mathrm{Ca}\left(\mathrm{mgL}^{-1}\right)$ & 78 & 106 & 71 & - & 86 & 141 & 66 \\
\hline $\mathrm{Mg}\left(\mathrm{mgL}^{-1}\right)$ & 26 & 25 & 19 & - & 37 & 56 & 69 \\
\hline $\mathrm{Na}\left(\mathrm{mgL}^{-1}\right)$ & 86 & 20 & 17 & - & 363 & 522 & - \\
\hline $\mathrm{K}\left(\mathrm{mgL}^{-1}\right)$ & 5.5 & 2.0 & 1.5 & - & 5.0 & 6.5 & 11.5 \\
\hline $\mathrm{NO}_{3}-\mathrm{N}\left(\mathrm{mgL}^{-1}\right)$ & 3 & 4 & 2 & 1 & 1.5 & 7 & \\
\hline $\mathrm{NO}_{3}^{-}\left(\mathrm{mgL}^{-1}\right)$ & 14.5 & 18.5 & 8.0 & 5.2 & 7.6 & 27.0 & 30.0 \\
\hline $\mathrm{NO}_{2}-\mathrm{N}\left(\mathrm{mgL}^{-1}\right)$ & 3 & 5 & & & & & \\
\hline $\mathrm{NH}_{3}-\mathrm{N}\left(\mathrm{mgL}^{-1}\right)$ & & 0.12 & 0.06 & 0.07 & 0.06 & 0.06 & 0.09 \\
\hline $\operatorname{COD}\left(\mathrm{mgL}^{-1}\right)$ & 30 & 13 & & & & & \\
\hline Cyanide $\left(\mathrm{mgL}^{-1}\right)$ & & 0.12 & 0.06 & 0.07 & 0.06 & 0.06 & 0.09 \\
\hline $\mathrm{CaCO}_{3}\left(\mathrm{mgL}^{-1}\right)$ & 180 & 230 & 181 & 140 & 215 & 280 & 160 \\
\hline
\end{tabular}

trations of nitrate were under the permissible limit from April 2002 to July 2002.

At well W-05 the $\mathrm{pH}$ and $\mathrm{EC}$ values were between 7.10-7.76 and 808-1124 $\mu \mathrm{S} / \mathrm{cm}$ respectively. Calcium concentration was $194.0 \mathrm{mgL}^{-1}$ in November 2001, which exceeded the permissible limit. Calcium concentration was not steady during the study period and it suddenly decreased to $50.0 \mathrm{mgL}^{-1}$ in December 2001. Although magnesium concentrations were about $20.0 \mathrm{mgL}^{-1}$, they decreased from 57.0 to $12.0 \mathrm{mgL}^{-1}$ in December 2002. Sodium concentrations were not constant. It was $265.0 \mathrm{mgL}^{-1}$ in November 2001, exceeding the limits; on the other hand, its value gradually decreased to $70.0 \mathrm{mgL}^{-1}$ in April 2002. Potassium concentrations were not constant with maximum concentration of $5.0 \mathrm{mgL}^{-1}$ in December 2001 and minimum concentration of $3.0 \mathrm{mgL}^{-1}$ in July $2002 . \mathrm{HCO}_{3}^{-}$concentrations were not constant during the study period with maximum concentration of $450.0 \mathrm{mgL}^{-1}$ in November 2001 and minimum concentration of $202.0 \mathrm{mgL}^{-1}$ in May 2002. According to their alkalinity values, the samples of well W-05 were very hard water in autumn 2001 and hard water in winter 2002 and summer 2002. Although $\mathrm{Cl}^{-}$concentration was $247.0 \mathrm{mgL}^{-1}$ in December 2001, it had lower values in other months. The concentrations of nitrate were under the permissible limit in July 2002.

At well W-06, calcium concentrations were not constant, because the earth's crust usually has major cations in high concentrations and the concentrations of major cations in groundwater samples change due to irrigation and/or rainfall. It is the use of this well for irrigation purposes that has caused the changes in calcium concentration. Magnesium concentrations were about $35.0 \mathrm{mgL}^{-1}$. Sodium concentrations were about $23.0 \mathrm{mgL}^{-1}$. $\mathrm{HCO}_{3}^{-}$and $\mathrm{Cl}^{-}$concentrations were not constant, but they were about 300.0 and $80.0 \mathrm{mgL}^{-1}$, respectively. Since W-06 is used for irrigation, the results were compared to irrigation water standards and concentrations of the parameters were found to be within permissible limits. According to results of the alkalinity tests, well W-06 was classified as hard water.

At well W-07, the $\mathrm{pH}$ and $\mathrm{EC}$ values were between 7.16-7.60 and 835-895 $\mu \mathrm{S} / \mathrm{cm}$ respectively. Calcium concentrations were about $120 \mathrm{mgL}^{-1}$, which were above the permissible limits. Magnesium and sodium concentrations were about 35.0 and $15.0 \mathrm{mgL}^{-1}$, respectively. Potassium concentrations were about $1.0 \mathrm{mgL}^{-1}$. $\mathrm{HCO}_{3}^{-}$concentrations were not constant during the study period with minimum concentration of $293.0 \mathrm{mgL}^{-1}$ and maximum concentration of $358.0 \mathrm{mgL}^{-1}$ in December 2001. Alkalinity tests show that well W-07 can be classified as hard water. The concentrations of nitrate exceeded the permissible limit. Maximum nitrate concentration was $65.0 \mathrm{mgL}^{-1}$ in February 2002 and the minimum nitrate concentration was $30 \mathrm{mgL}^{-1}$ in October 2001 .

At well W-08, the $\mathrm{pH}$ and $\mathrm{EC}$ values were between $7.30-7.50$ and $726-867 \mu \mathrm{S} / \mathrm{cm}$ respectively. Calcium concentrations were about $110 \mathrm{mgL}^{-1}$, exceeding the permissible limits. Magnesium and sodium concentrations respectively were about 30.0 and $20.0 \mathrm{mgL}^{-1}$, 
within TS 266. $\mathrm{HCO}_{3}^{-}$concentrations were not constant during the study period. Alkalinity results show that $\mathrm{W}-08$ had hard water. $\mathrm{Cl}^{-}$concentrations were about $40.0 \mathrm{mgL}^{-1}$, which was within permissible limits. The concentrations of nitrate exceeded the permissible limit in March and July 2002. The maximum nitrate concentration was $48.0 \mathrm{mgL}^{-1}$ in March 2002.

At well $\mathrm{W}-09$, the $\mathrm{pH}$ and $\mathrm{EC}$ values were between 7.30-7.51 and 682-754 $\mu \mathrm{S} / \mathrm{cm}$ respectively. Calcium, magnesium, sodium, potassium concentrations of the sample were within the permissible limits. $\mathrm{HCO}_{3}^{-}$ concentrations were not constant during the study period. $\mathrm{Cl}^{-}$concentrations were about $35.0 \mathrm{mgL}^{-1}$, within permissible limits. The concentrations of nitrate were under the permissible limit.

At Fetrek Creek (sample S-01), all parameters were not constant. Every month, values of each parameter were different from the previous month. This is because this creek was dry and industrial wastewaters had been discharged into the creek before April 2002. In rainy seasons, concentration of all the parameters in the wells as well as the creek decreased because of dilution by rain. The amount of rainfall was high in autumn 2001, winter 2002, and April 2002.

Chloride concentrations were constant for all samples except at wells W-02, W-05, and W-06. The chloride concentration of well W-05 decreased, but the chloride concentration of well W-06 increased with time. The permissible limit for chloride is $250 \mathrm{mgL}^{-1}$. All samples did not exceed the limit except well W02 , in which the chloride concentration changed every month of the monitoring period. Well W-02 is located among textile manufacturers near Fetrek Creek. Therefore, it can be stated that the well is under threat of contamination by Fetrek Creek and/or industry.

Bicarbonate concentrations of the samples changed gradually. This means that $\mathrm{HCO}_{3}^{-}$concentrations of all samples increased or decreased in the same month because of rainfall. In groundwater samples only bicarbonate alkalinity exists. During analysis of the alkalinity, the color of the samples did not change with the addition of phenolphthalein. This means that the samples had only bicarbonate alkalinity and there was no carbonate or hydroxide alkalinity.

At well W-02 sodium ion concentration suddenly increased in January 2002. The well is in the field of a textile factory near the Fetrek Creek. The high values of sodium can be explained by leakage from Fetrek
Creek. Chloride and sodium ion concentrations changed at the same time. This can be explained by leakage of $\mathrm{NaCl}$.

According to TS 266, calcium ion concentration should not exceed $100 \mathrm{mgL}^{-1}$. Calcium ion concentrations exceed the permissible limit at wells $\mathrm{W}-01$, W-02, W-03, W-06, W-07, W-08, and W-09. Calcium cation concentrations of the samples were constant during the monitoring period except in well W-05, where calcium concentration suddenly decreased from 194 to $49.40 \mathrm{mgL}^{-1}$ in December 2001 and did not stay stable. At well $\mathrm{W}-05, \mathrm{HCO}_{3}^{-}$concentration changed by the same amount as calcium. This implies that groundwater of well W-05 interacts with calcite, which is permeable rock allowing the contamination to reach the groundwater.

At well W-05, sodium ion concentration was more than $175 \mathrm{mgL}^{-1}$ in November 2001, which is a permissible limit according to TS 266. However, the concentration of sodium decreased gradually every month until July 2002. W-05 is in field of a food factory near Fetrek Creek. Tomatoes are dried and tinned in the factory. The sudden changes can be explained by the leakage of $\mathrm{NaCl}$ from factory storage land or from Fetrek Creek. Chloride and sodium ion concentrations changed in the same months. Tomato is dried in the summer and $\mathrm{NaCl}$ is used in the drying applications beyond the boundaries of the factory. These results show that $\mathrm{NaCl}$ infiltrated the groundwater in summer and autumn 2001.

The permissible limit for magnesium ion is $50 \mathrm{mgL}^{-1}$. At well W-02 magnesium ion concentrations were over the permissible limit. Potassium is less soluble in water compared to other cations and the concentrations of potassium were under the permissible limit. Cyanide concentrations of the samples ranged between 0 and $0.005 \mathrm{mgL}^{-1}$ and were not constant during the monitoring study. This can be explained by the leachate of wastewaters from Fetrek Creek.

Average chemical characteristics of water compositions (see Table 2), on the basis of major ion concentrations, were evaluated on a Piper and Schoeller diagram (Figs. 3 and 4). When concentrations of the elements are evaluated, it can readily be seen that there are three types of water in Torbal Basin. First, water that is relatively rich in $\mathrm{Ca}-\mathrm{HCO}_{3}$ (calcium-bicarbonate type), as seen in $\mathrm{W}-01, \mathrm{~W}-03$, $\mathrm{W}-04, \mathrm{~W}-06, \mathrm{~W}-07, \mathrm{~W}-08$ and $\mathrm{W}-09$. In the second 
Table 2 Average major anion and cation in water around Torbalı Basin (average data from November 2001 to July 2002 , $\mathrm{SO}_{4}$ calculate mathematically)

\begin{tabular}{|c|c|c|c|c|c|c|c|c|c|c|}
\hline Sample number & W-01 & W-02 & W-03 & W-04 & W-05 & W-06 & W-07 & W-08 & W-09 & S-01 \\
\hline $\mathrm{HCO}_{3}\left(\mathrm{mgL}^{-1}\right)$ & 263.4 & 255 & 266 & 261 & 278 & 293.6 & 314.4 & 303.7 & 270.6 & 251.1 \\
\hline $\mathrm{SO}_{4}\left(\mathrm{mgL}^{-1}\right)$ & 166 & 132 & 140 & 107 & 110 & 109 & 137 & 138 & 98 & 643 \\
\hline $\mathrm{Cl}\left(\mathrm{mgL}^{-1}\right)$ & 37.93 & 326 & 51.1 & 39.1 & 141 & 92.16 & 51.13 & 46.08 & 39.25 & 208.5 \\
\hline $\mathrm{Ca}\left(\mathrm{mgL}^{-1}\right)$ & 101.6 & 159 & 106 & 92 & 80.1 & 110.2 & 116.8 & 109.8 & 95.88 & 282.49 \\
\hline $\mathrm{Mg}\left(\mathrm{mgL}^{-1}\right)$ & 33.5 & 50.4 & 32.4 & 29 & 23 & 36.6 & 34.22 & 34.4 & 26.22 & 31.125 \\
\hline $\mathrm{Na}\left(\mathrm{mgL}^{-1}\right)$ & 15.75 & 64.1 & 16.8 & 14 & 111 & 24.2 & 17.88 & 18.6 & 14.11 & 150.28 \\
\hline $\mathrm{K}\left(\mathrm{mgL}^{-1}\right)$ & 1.75 & 2.06 & 1.06 & 0.87 & 3.88 & 1 & 1.27 & 1.2 & 1.16 & 4.81 \\
\hline
\end{tabular}

type, the water is relatively rich in $\mathrm{Ca}-\mathrm{CI}$ (calciumsodium-chlorite type), which can be seen in W-02 and $\mathrm{W}-05$. The third type of water is relatively rich in $\mathrm{Ca}-\mathrm{SO}_{4}$ (calcium-sulphate type), which can be seen in $\mathrm{S}-01$.

All samples were analyzed to investigate heavy metals. Inductively coupled plasma-atomic emission spectrometry was used to detect $\mathrm{Cd}, \mathrm{Cr}, \mathrm{Cu}, \mathrm{Pb}$ and Zn. During the study period, heavy metals could not be detected except in well W-05 in November 2001. Zinc concentration in well W-05 was nearly $7 \mathrm{mgL}^{-1}$, which is higher than the permissible limit. However, the concentration of zinc decreased in other months. Lead concentration in well W-05 was $0.02 \mathrm{mgL}^{-1}$ in November 2001, more than the permissible lead concentration of $0.01 \mathrm{mgL}^{-1}$. However, like zinc, lead concentration decreased in other months. The

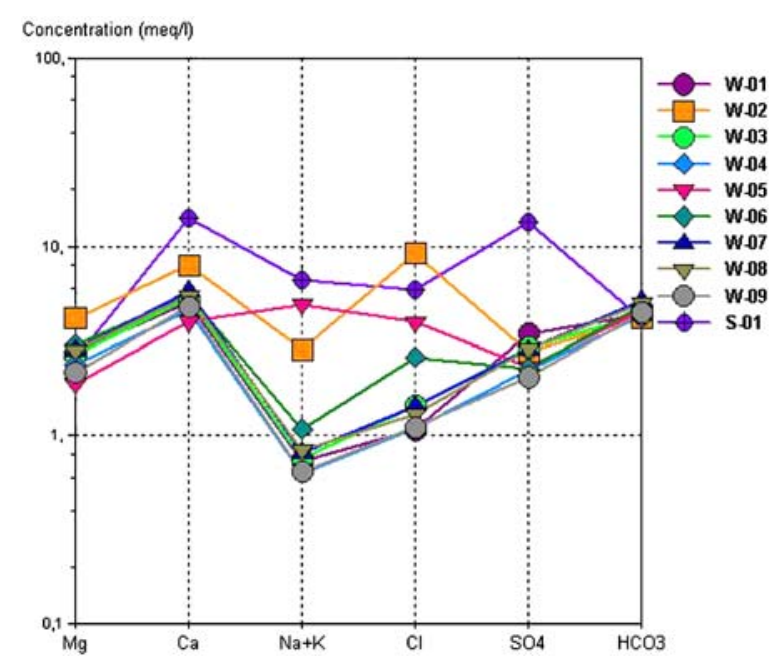

Fig. 3 Chemical analysis of water of the study are plotted on Schoeller diagrams other parameters of well W-05 also had higher values in November 2001. All of the parameters of well $\mathrm{W}-05$ were not constant during the monitoring period. This situation can be explained by sudden discharges of wastewater into Fetrek Creek infiltrating well W-05 close by food industry (see Fig. 2).

According to the TS 266, the permissible limit for nitrate is $45 \mathrm{mgL}^{-1}$. $\mathrm{NO}_{3}^{-}$concentrations of the samples were under the permissible limit except at wells W-07 and W-08. Nitrate concentrations of all the samples decreased from October 2001 to April 2002. This corresponds to the rainy season and there is no agricultural activity during this period. This means that there was no source for nitrate contamination. During this period rain may have caused some dilution, resulting in decreased nitrate concentration. On the other hand, the results show that nitrate concentration increased in the summer, clear evidence of agricultural contamination due to manure containing nitrate compounds being applied during the summer. Irrigation may have caused leakage of the nitrate.

The concentration of $\mathrm{NH}_{3}-\mathrm{N}$ must not exceed $0.02 \mathrm{mgL}^{-1}$. According to the TS 266, nitrite concentration must be less than $0.05 \mathrm{mgL}^{-1}$. The values of nitrite nitrogen and ammonia nitrogen concentrations in the samples were higher than the permissible limits. This can be explained by the leakage of sewage to groundwater.

Fecal and total coliform microorganisms were analyzed at well W-09 which is one of the sources for municipal water usage. Microorganisms were not found in the well W-09 in January 2002.

Chemical oxygen demand (COD) concentration in the samples decreased in the rainy seasons. COD analysis was only carried out up to February 2002 due to technical difficulties. COD concentrations of the 
Fig. 4 Chemical analysis of water of the study are plotted on Piper diagram

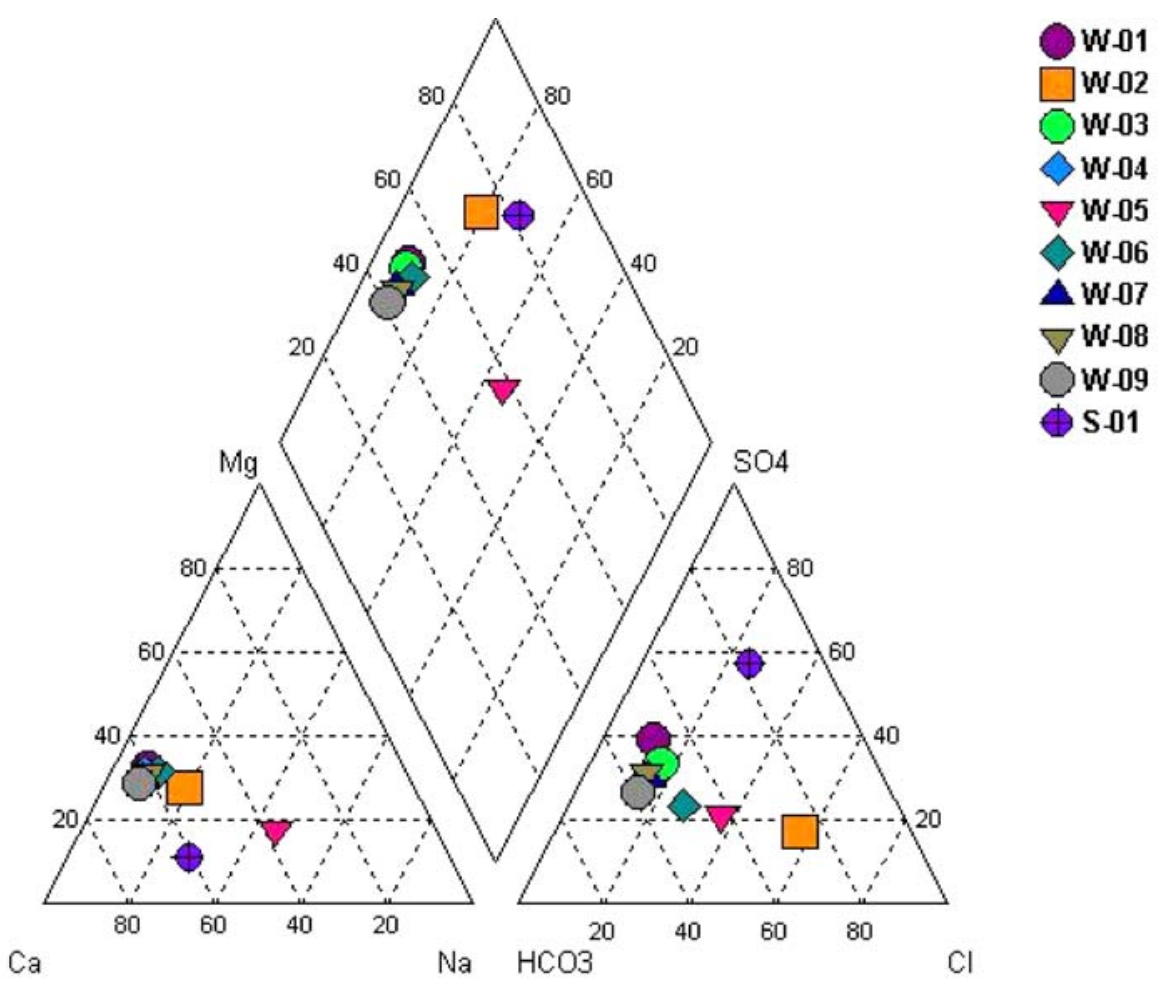

wells W-02 and W-05 were higher than $10 \mathrm{mgL}^{-1}$ in October 2001 and February 2002. In the wells, concentrations of the other parameters were high in October 2001. This can also be explained by leakage from Fetrek Creek.

\section{Conclusions and recommendations}

In the study area, since industrial activity is growing, the population of the region is also increasing. Accordingly, the demand for water is growing and consequently water depletion increases. There are many factories in the region, nearly all of which had discharged their wastewaters into Fetrek Creek up until April 2002. The waste disposal site of the region which is near a farm has not been designed properly, so contaminants may have been infiltrating the groundwater.

As a result of interactions between groundwater and geological materials, groundwater in Torbalı region has a bicarbonate alkalinity and is classified as hard water.

Agricultural contamination due to nitrates was determined in the region. The nitrate concentration of the samples increased in summer because of agricultural activities. Manures which include nitrogen compounds were also used in the summer period and therefore irrigation had caused leachate of nitrate. The wrongful use of nitrate-containing compounds in agriculture needs to be controlled, especially since the toxicity of nitrate causes methemoglobinemia in infants.

Nitrite and ammonia concentrations were found to be above drinking water standard safety limits. Also, total coliform was detected in some of the wells according to monitoring studies conducted by the Torbalı Directorate of Health.

Fetrek Creek is dry in summer and the creek bed was used by industry as a discharge point for wastewaters, a practice that continued up until April 2002. Chemical oxygen demand concentrations were higher than the permissible limits in some months during the monitoring period. This is because industrial wastewaters might have infiltrated groundwater from Fetrek Creek. Therefore, the discharge points of factories need to be strictly controlled.

Groundwater conservation areas should be ascertained as is the case for Torbalı Watershed. The consumption of manure and chemicals (insecticides, herbicides, etc.) should be limited in agricultural activities. The permissible limits must not be exceeded. 
Leakage from solid waste disposal areas threatens groundwater resources. There are agricultural activities and wells around the solid waste disposal site in Torbal1. Therefore, leakage should be prevented.

According to the Control of Water Pollution Regulation (Official Gazette 1988), wells must be prevented from becoming polluted. Conservation areas of the wells must be hedged and they should be strictly controlled to prevent contamination. No settlement must exist nearby the conservation areas. Infiltration of chemicals, wastewaters, etc. must be prevented. The solid waste disposal area must be designed to prevent infiltration. According to the regulation, agricultural chemicals must be easily degradable in nature and must not accumulate in the human body when groundwater is used for drinking.

More knowledge about well construction features, conditions, and location of the well relative to water distribution, storage or treatment systems is needed to evaluate the suitability of a well for usage. Information about existing wells in Torbalı has not yet been gathered for the whole study area. Groundwater vulnerability mapping studies also need to be carried out in the area.

Surface water and groundwater can be used conjunctively. There are many advantages of groundwater storage compared to surface storage. Conjunctive use of water is cheaper than dams and reservoirs operated separately.

Protection of groundwater quantity and quality can be accomplished by controlling potential contaminant sources and by managing land use in primary recharge areas. Using knowledge of local geology and groundwater flow directions, estimates can be made of the land areas contributing recharge to a particular well or to an aquifer as a whole. Controls can then be established to ensure appropriate land use and chemical practices within the recharge areas. The best protection is provided through land acquisition. Agricultural activities and industrial applications should be controlled over the aquifer.

In this study, microbiological assessment was not applied for all the wells, therefore the question of microbiological contamination requires further studies, which may also determine pesticide contamination in the region. Sudden discharges should also be determined using advanced monitoring methods. In this study, samples were collected monthly for 10 months. However, there is a need for monitoring studies applied to the study area for a much longer duration. Therefore, this study should not be the last one of the region.

\section{References}

Afsin, M. (1997). Hydrochemical evolution and water quality along the groundwater flow path in the Sandikli Plain, Afyon, Turkey. Environmental Geology, 31(3-4), 221-230.

Asaroğlu, M., Sunlu, U., \& Kaymakç1, A. (1999). Investigating quality criteria of water resources in regions of İzmir. Proceeding of Izmir Water Congress, İzmir-1999 (in Turkish).

Aslan, S,., Türkman, A., Övez, B., Yüksel, M., \& Alyanak, İ. (2001). Determination of groundwater contamination in Aegean Region, Urla and Menemen Vicinity. ÇEVJEO'2001 proceedings, İzmir-2001,125-131 (in Turkish).

AWWA (1995). Chemical oxygen demand, argentometric method. In Standard Methods for the Examination of Water and Wastewater (pp. 5-12). Washington: American Public Health Association 4(49).

Baba, A., \& Ayyıldız, O. (2006). Urban groundwater pollution in Turkey, a national review of urban groundwater quality issues, urban groundwater management and sustainability. In J. H. Tellam, M. O. Rivette, R. G. Israfilov (Eds.), IV. Earth and Environmental Sciences, NATO Science Series, Springer (93-110).

Baba, A., Birsoy, Y. K., Ensari, E., Andic, T., \& Baykul, A. (2001). Izmir city's groundwater quality and pollution. ÇEVJEO'2001 Proceedings, March 21-23, Izmir, 49-158.

Baba, A., \& Sözbilir, H. (2001). Geology and groundwater quality of Kemalpaşa - Torbalı Plain, West Anatolia. ÇEVJEO’2001 Proceedings, İzmir-2001, 133-148.

Belgiorno, V., \& Napoli, R. M. A. (2000). Groundwater quality monitoring. Water Science and Technology, 42(1-2), 37-41.

Ekmekçi, M., \& Günay, G. (1997). Role of public awareness in groundwater protection. Environmental Geology, 30(1-2), 81-87.

Eryurt, A., \& Sekin, Y. (2001). Seasonal changes in hardness and nitrate of groundwater in the region of Manisa. Proceedings of ÇEVJEO'2001 Proceedings, İzmir-2001, 187-193 (in Turkish).

Hu, K., Huang, Y., Li, H., Chen, B. L. D., \& White, R. E. (2005). Spatial variability of shallow groundwater level, electrical conductivity and nitrate concentration, and risk assessment of nitrate contamination in North China Plain. Environment International, 31(6), 896-903.

Kaçaroğlu, F. (1999). Review of groundwater pollution and protection in karst areas. Water, Air, and Soil Pollution, $113(1-4), 337-356$.

Kaçaroğlu, F., \& Günay, G. (1997). Groundwater nitrate pollution in an alluvium aquifer, Eskisehir Urban area and its vicinity, Turkey. Environmental Geology, 31(3-4), $178-184$.

Kampbell, D. H., An, Y.-J., Jewell, K. P., \& Masoner, J. R. (2003). Groundwater quality surrounding Lake Texacoma during short-term drought conditions. Environmental Pollution, 125(2), 183-191. 
Karagüzel, R., Scholz, R., \& Ebel, B. (1999). Hydrogeological investigation of Antalya basin concerning the future domestic water needs of Antalya City (Turkey). Environmental Geology, 38(2), 159-167.

Karlık, G., \& Kaya, M. A. (2001). Investigation of groundwater contamination using electric and electromagnetic methods at an open waste-disposal site: A Case Study from Isparta, Turkey. Environmental Geology, 40(6), 725-731.

Kumbur, H., Arslan, H., \& Özer, Z. (2001). Investigation of contamination of drinking and usage water resources of Icel. I. Turkish Water Congress Proceedings, İstanbul-2001 (in Turkish).

Leung, C. M., \& Jiao, J. J. (2006). Heavy metal and trace element distributions in groundwater in natural slopes and highly urbanized spaces in Mid-Levels area, Hong Kong. Water Research, 40(4), 753-767.

Official Gazette (1988). Issue 19919, Controlling of water pollution regulation, Ankara.

Özçelik, Ș., \& Sariz, K. (2001). Eskișehir ovası yeraltısuyu kirliliğinde 1995 sonrası olumlu gelișmeler. Proceedings of ÇEVJEO'2001 Proceedings, İzmir-2001, 231-246 (in Turkish).

Polat, M., \& Y1lmaz, N. (2001). Antalya havzasinda yeraltısu kaynaklarında kirliliğin belirlenmesi ve koruma stratejileri. Proceedings of ÇEVJEO'2001 Proceedings, İzmir-2001, 247-253 (in Turkish).

Reijnders, H. F. R., Drecht van, G., Prins, H. F., \& Boumans, L. J. M. (1998). The quality of the groundwater in Netherlands. Journal of Hydrology, 207, 179188 .
Şimșek, C., \& Filiz, Ș. (2001). Investigation hydrogeological of Torbalı Basin. Proceedings of ÇEVJEO'2001 Proceedings, İzmir-2001, 275-283 (in Turkish).

Soltan, M. E. (1998). Characterization, and evaluation of some ground water samples in upper Egypt. Chemosphere, 37 (4), 735-745.

Tariq, M. I., Afzal, S., \& Hussain, I. (2004). Pesticides in shallow groundwater of Bahawalnagar, Muzafargarh, D.G. Khan and Rajan Pur districts of Punjab, Pakistan. Environment International, 30(4), 471-479.

Turan, M., Alp, K., Övez, S., Bayhan, H., \& Topacik, D. (2001). Treatability and characterization of groundwater used as process water in Thrace Region. ÇEVJEO'2001 Proceedings, İzmir-2001, 409-415.

Turkish Standards (TS) (2005). Water intended for human consumption, Standard, Ankara, Turkey.

Umar, R., \& Ahmad, M. S. (2000). Groundwater quality in parts of central Gang Basin, India. Environmental Geology, 39 (6), 673-678.

US.EPA. (1993). Standard methods for the examination of water and wastewater, US. Environmental Protection Agency (US.EPA). American Publish Health Assoc.

Wakida, F. T., \& Lerner, D. N. (2005). Non-agricultural sources of groundwater nitrate: a review and case study. Water Research, 39(1), 3-16.

Yılmaz, Y., Genç, Ș. C., Güner, F., Bozcu, M., Yılmaz, K., Korack, Z., et al. (2000). When did the Western Anatolia grabens begin to develop. In E. Bozkurt, S. A Winchester, and J. D. A. Piper (Eds.), Tectonics and magmatism in Turkey and the surrounding area, Geological Soc. London, Special Pub., 173 (pp. 353-384). 